\title{
A novel approach for transtunnel puncture in a patient with atrial tachyarrhythmias and Fontan circulation
}

\author{
Christian Sohns $^{1} \mathbb{1} \cdot$ Leonard Bergau $^{1} \cdot$ Philipp Sommer $^{1} \cdot$ Stephan Molatta ${ }^{1,2}$
}

Received: 31 March 2021 / Accepted: 3 August 2021 / Published online: 24 August 2021

(c) The Author(s) 2021

In patients with Fontan circulation, lateral intraatrial tunnel, and spontaneously closed lateral tunnel fenestration, transtunnel puncture (TP) might be required to perform ablation in the functional atrium (LA). We report of a patient with drug-refractory atrial arrhythmias and Fontan circulation. This case demonstrates a novel multimodal approach for TP using a steerable sheath (ViziGo, medium curve, Biosense Webster) allowing its visualization inside the 3D-mapping system to avoid the need of fluoroscopy. Intracardiac echography (ICE) was utilized in conjunction with merging the $3 \mathrm{D}$ reconstruction from previous magnetic resonance imaging-guided angiography to visualize the complex anatomy in real time and to rule out LA thrombus formation. TP was performed with a Brockenbrough needle BRK1. TP followed a stepwise approach: First, we performed 3D reconstruction of the Fontan tunnel in $\boldsymbol{A}$ RAO $30^{\circ}$ and $\boldsymbol{B}$
LAO $60^{\circ}$ projection. Second, we determined the puncture site (turquoise mark; white arrow) using the visualization of the steerable sheath, a force-sensing catheter (Thermocool SmartTouch SF, Biosense-Webster), and ICE. There was no evidence for a residual fenestration of the tunnel. The sheath was continuously visualized during TP and carefully advanced to the LA ( $\boldsymbol{C}$ RAO $30^{\circ}$ projection; $\boldsymbol{D}$ LAO $60^{\circ}$ projection). After access to the LA, $\boldsymbol{E}, \boldsymbol{F}$ pulmonary vein isolation was performed with ablation index-guided $\mathrm{RF}$ applications (red tags around the ipsilateral pulmonary veins in $\boldsymbol{E}, \boldsymbol{F})$. The clinical atrial tachycardia was induced, mapped, and ablated with substrate modification at the anterior atrial wall in close relationship to the Fontan tunnel (red tags in $\boldsymbol{E}$; PA, pulmonary arteries; IVC, inferior vena cava; LIPV, left inferior pulmonary vein). There were no procedure-related complications.
Christian Sohns

Christian.Sohns@ruhr-uni-bochum.de

1 Clinic for Electrophysiology, Herz- Und Diabeteszentrum NRW, Ruhr-Universität Bochum, Georgstrasse 11, 32545 Bad Oeynhausen, Germany

2 Center for Congenital Heart Disease/Pediatric Heart Center, Herz- Und Diabeteszentrum NRW, Ruhr-Universität Bochum, Georgstrasse 11, 32545 Bad Oeynhausen, Germany 


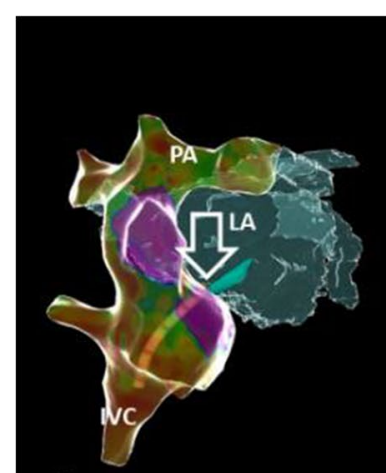

A

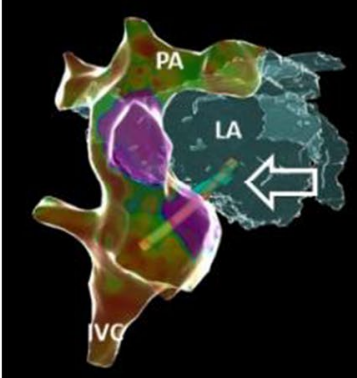

C

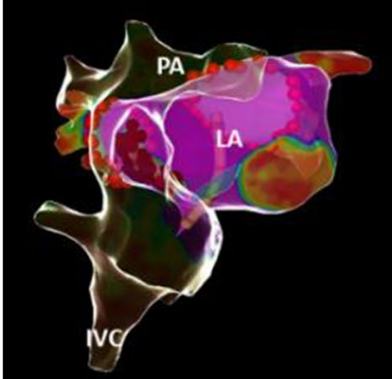

E

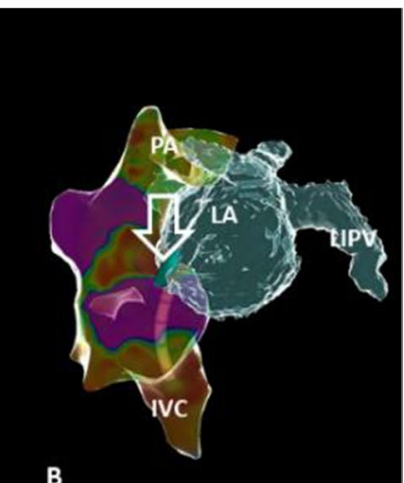

B

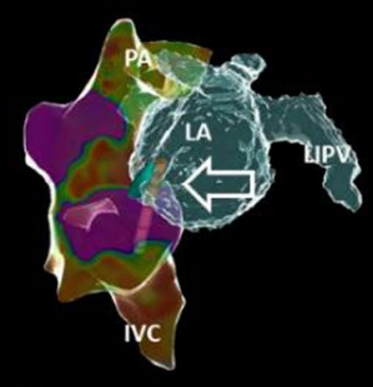

D

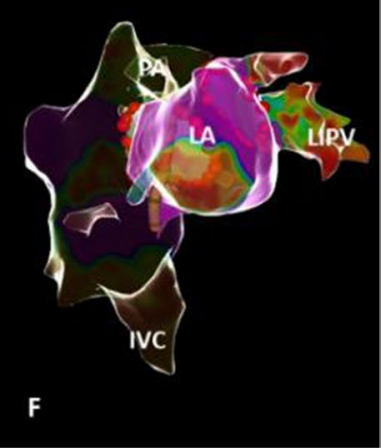

Acknowledgements We acknowledge support by the DFG Open Access Publication Funds of the Ruhr-Universität Bochum.

Funding Open Access funding enabled and organized by Projekt DEAL.

\section{Declarations}

Conflict of interest The authors declare no competing interests.

Open Access This article is licensed under a Creative Commons Attribution 4.0 International License, which permits use, sharing, adaptation, distribution and reproduction in any medium or format, as long as you give appropriate credit to the original author(s) and the source, provide a link to the Creative Commons licence, and indicate if changes were made. The images or other third party material in this article are included in the article's Creative Commons licence, unless indicated otherwise in a credit line to the material. If material is not included in the article's Creative Commons licence and your intended use is not permitted by statutory regulation or exceeds the permitted use, you will need to obtain permission directly from the copyright holder. To view a copy of this licence, visit http://creativecommons.org/licenses/by/4.0/.

Publisher's note Springer Nature remains neutral with regard to jurisdictional claims in published maps and institutional affiliations. 